\title{
ROTATION AND SURFACE ABUNDANCE PECULIARITIES IN A-TYPE STARS
}

\author{
Yoichi TAKeda ${ }^{1}$, Inwoo $\mathrm{HAN}^{2}$, Dong-Il KAnG ${ }^{3}$, Byeong-Cheol LeE ${ }^{2,4}$, And Kang-Min Kim ${ }^{2}$ \\ ${ }^{1}$ National Astronomical Observatory of Japan, 2-21-1 Osawa, Mitaka, Tokyo 181-8588, Japan \\ E-mail: takeda.yoichi@nao.ac.jp \\ ${ }^{2}$ Korea Astronomy and Space Science Institute, 61-1 Whaam-dong, Youseong-gu, Taejon 305-348, Korea \\ E-mail: iwhan@kasi.re.kr,bclee@boao.re.kr,kmkim@boao.re.kr \\ 3 Gyeongsangnamdo Institute of Science Education, 75-18 Gajinri, Jinsungmyeon, \\ Jinju, Gyeongnam 660-851, Korea \\ E-mail: kangdongil@gmail.com \\ ${ }^{4}$ Department of Astronomy and Atmospheric Sciences, Kyungpook National University, Daegu 702-701, Korea \\ (Received June 24, 2008; Accepted August 25, 2008)
}

\begin{abstract}
In an attempt of clarifying the connection between the photospheric abundance anomalies and the stellar rotation as well as of exploring the nature of "normal A" stars, the abundances of seven elements (C, O, Si, Ca, Ti, Fe, and $\mathrm{Ba}$ ) and the projected rotational velocity for 46 A-type field stars were determined by applying the spectrum-fitting method to the high-dispersion spectral data obtained with $\mathrm{BOES}$ at BOAO. We found that the peculiarities (underabundances of $\mathrm{C}, \mathrm{O}$, and $\mathrm{Ca}$; an overabundance of $\mathrm{Ba}$ ) seen in slow rotators efficiently decrease with an increase of rotation, which almost disappear at $v_{\mathrm{e}} \sin i \gtrsim 100 \mathrm{~km} \mathrm{~s}^{-1}$. This further suggests that stars with sufficiently large rotational velocity may retain the original composition at the surface without being altered. Considering the subsolar tendency (by several tenths dex below) exhibited by the elemental abundances of such rapidly-rotating (supposedly normal) A stars, we suspect that the gas metallicity may have decreased since our Sun was born, contrary to the common picture of galactic chemical evolution.
\end{abstract}

Key words : stars: abundances — stars: atmospheres — stars: early-type stars: chemically-peculiar — stars: rotation

\section{INTRODUCTION}

Since unevolved A-type stars on (or near to) the upper main-sequence have masses around $\sim 2 M_{\odot}$, their surface abundances may retain information of the composition of the past galactic gas $\left(\lesssim 10^{9} \mathrm{yr}\right.$ ago) from which they formed; this would provide us with an important opportunity to investigate the "recent" chemical evolution history of the Galaxy. However, such a study using A stars as a probe of late-time history of chemical evolution has rarely been done in spite of its potential significance* in contrast to the case of old-time history where a number of researches (using longer-lived $\mathrm{F}-\mathrm{G}-\mathrm{K}$ dwarfs) are available. This is related to the fact that a large fraction of them are rapid rotators (typically $v_{\mathrm{e}} \sin i \sim 100-200 \mathrm{~km} \mathrm{~s}^{-1}$ on the av-

Corresponding Author: Y. Takeda

* For example, Takeda, Sato, \& Murata (2008) found in their extensive study of late-G giants (also having masses around $\sim 2 M_{\odot}$; evolved counterparts of A dwarfs) that their metallicities show an appreciable diversity as large as $\sim 1 \mathrm{dex}$ $(-0.8 \lesssim[\mathrm{Fe} / \mathrm{H}] \lesssim+0.2)$ with a subsolar trend on the average (cf. Fig. 14 therein), from which they argued that some special event (like a mixing of metal-poor primordial gas caused by infall) might have occurred $\sim 10^{9}$ yr ago. However, before making any speculation, it is important to confirm whether such a trend is also observed in their progenitors in the upper main-sequence. erage; see, e.g., Royer et al. 2002a, b) whose spectra are technically difficult to analyze because lines are broad and smeared out, while sharp-lined slow rotators easy to handle tend to show abundance anomalies (chemically peculiar stars or CP stars).$^{\dagger}$ As a matter of fact, most spectroscopic analyses of A stars have focused on sharp-lined ones with $v_{\mathrm{e}} \sin i \lesssim 50 \mathrm{~km} \mathrm{~s}^{-1}$. For this reason, nobody could be sure whether the result obtained for star classified as "normal A" really reflects the initial composition free from any peculiarities.

Therefore, in order to make a further step forward, it is requisite to challenge abundance determinations for "unbiased" sample of A-type stars in general (i.e., without sidestepping rapidly rotating ones), which inevitably requires an application of the spectrum synthesis technique, since reliably measuring the equivalent widths of individual spectral lines is almost hope-

\footnotetext{
$\dagger$ Although many things are left unresolved concerning the origin and nature of the CP phenomena, it is widely considered (at least in the qualitative sense) that the chemical segregation in the stable atmosphere is responsible for the abundance anomalies at the surface: i.e., an element becomes over- or under-abundant depending on the balance of upward radiation force and the downward gravitational force. In this case, rotation would act against an efficient built-up of such anomalies because it enhances mixing of outer stellar layers via shear instability or meridional circulation.
} 
less for rapid rotators. Admittedly, while such trials of determining abundances from spectra of A dwarfs including broad-lined ones have recently emerged thanks to the improvement in the method of analysis as well as the data quality, their interests are mainly directed to objects of specific types; e.g., Vega-like stars (Dunkin et al. 1997) or $\lambda$ Bootis stars (Andrievsky et al. 2002) or open-cluster stars (Takeda \& Sadakane 1997; Varenne \& Monier 1999; Gebran et al. 2008; Gebran \& Monier 2008; Fossati et al. 2008). Namely, a systematic study attempting to clarify the characteristics of normal field A-type stars in general, especially in terms of their abundance-rotation connection, seems to have been rarely attempted. To our knowledge, only one such study is Lemke's $(1990,1993)$ determinations of $\mathrm{C}$ and Ba abundances for some 20 rapidly-rotating A stars with $v_{\mathrm{e}} \sin i$ up to $\sim 200 \mathrm{~km} \mathrm{~s}^{-1}$, which however appear to be still insufficient and inconclusive as judged from his adopted method of approach as well as the number of elements studied.

Considering this situation, we decided to revisit this problem in our own manner based on the highdispersion spectral data of $\sim 50$ A-type stars in a wide range of $v_{\mathrm{e}} \sin i\left(0-300 \mathrm{~km} \mathrm{~s}^{-1}\right)$ obtained with BOES at BOAO, while applying the automatic spectrum fitting algorithm (Takeda 1995) which efficiently enables determinations of the abundances (for selected six elements of $\mathrm{C}, \mathrm{O}, \mathrm{Si}, \mathrm{Ti}, \mathrm{Fe}$, and $\mathrm{Ba}$ ) even for rapid rotators showing considerably merged spectra. Our ultimate aim is to clarify the following questions of interest:

- (1) Is there any systematic rotation-dependent tendency between slow and rapid rotators in terms of the abundance anomaly? If so, what is the critical value of $v_{\mathrm{e}} \sin i$, above which stars may be regarded as normal? - (2) What would the abundance characteristics of "normal A-type stars" like, which we may consider as retaining the composition of the galactic gas from which they formed?

We will show that reasonable answers to these points are provided from this study (cf. Sect. V).

\section{OBSERVATIONAL DATA}

We selected 46 apparently bright $(V \lesssim 5$ mag) Atype stars (including Am stars ${ }^{\ddagger}$ ) as our targets, which are listed in Table 1. Figure 1 shows the plots of these stars on the theoretical HR diagram, where we can see

$¥$ Although we excluded SrCrEu-type Ap stars (many are known to have magnetic fields) as well as $\lambda$ Boo stars (dust-gas separation process may be responsible for their metal-deficient trend), which show outstandingly large abundance peculiarities, Am stars (metallic-line stars which show comparatively mild anomaly) were included in our list (as was done by Takeda \& Sadakane 1997), since otherwise we can not realize a statistically meaningful sample of stars with a wide range of $v_{\mathrm{e}} \sin i$; i.e., the number of stars with small $v_{\mathrm{e}} \sin i$ which are not classified as Am is too small. (Since Am peculiarity is considered to be a natural phenomenon accompanied by slow rotation, Am stars would rather be interpreted as "ordinary" slowly-rotating A stars).

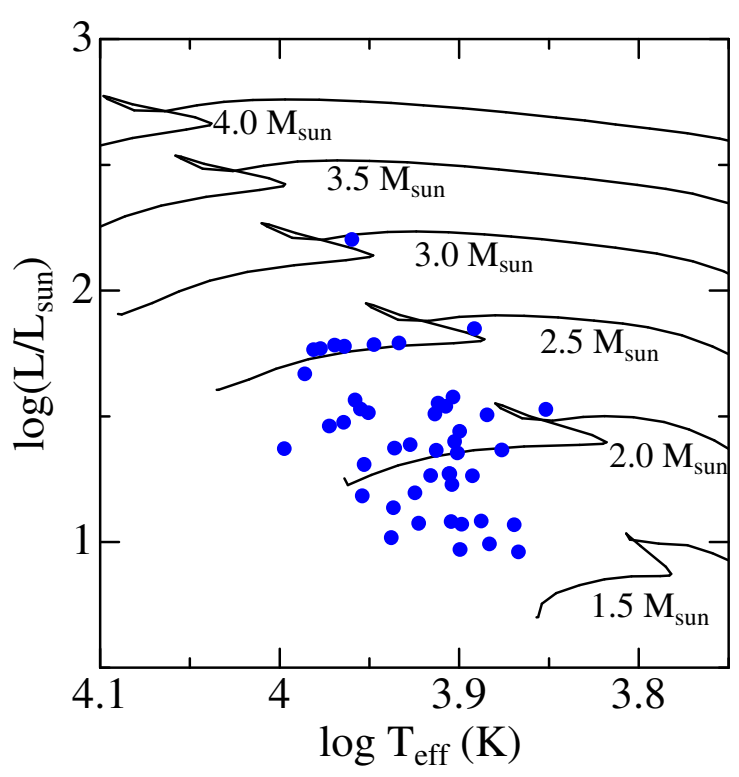

Fig. 1.- Plots of 46 program stars on the theoretical HR diagram $\left(\log \left(L / L_{\odot}\right)\right.$ vs. $\left.\log T_{\text {eff }}\right)$, where the bolometric luminosity $(L)$ was evaluated from the apparent visual magnitude with the help of Hipparcos parallax (ESA 1997) and Flower's (1996) bolometric correction. Theoretical evolutionary tracks corresponding to the solar metallicity computed by Girardi et al. (2000) for six different initial masses are also depicted for comparison.

that their masses are in the range of $1.5 M_{\odot} \lesssim M \lesssim$ $3 M_{\odot}$.

The observations were carried out on 2008 January 14-16 by using BOES (Bohyunsan Observatory Echelle Spectrograph) attached to the $1.8 \mathrm{~m}$ reflector at Bohyunsan Optical Astronomy Observatory. Using $2 \mathrm{k} \times 4 \mathrm{k}$ CCD (pixel size of $15 \mu \mathrm{m} \times 15 \mu \mathrm{m}$ ), this echelle spectrograph enabled us to obtain spectra of wide wavelength coverage (from $\sim 3700 \AA$ to $\sim 10000 \AA$ ) at a time. We used $200 \mu \mathrm{m}$ fiber corresponding to the resolving power of $R \simeq 45000$. The integrated exposure time for each star was typically $\sim 10-20 \mathrm{~min}$ on the average.

The reduction of the echelle spectra (bias subtraction, flat fielding, spectrum extraction, wavelength calibration, and continuum normalization) was carried out with the software developed by Kang et al. (2006). For all 46 targets, we could accomplish $\mathrm{S} / \mathrm{N}$ ratio of $\sim 300-500$ at the $6150 \AA$ region (on which we placed the largest weight on abundance determination; cf. Sect. IV).

\section{ATMOSPHERIC MODELS}

The effective temperature $\left(T_{\text {eff }}\right)$ and the surface gravity $(\log g)$ of each program star were determined 
TABLE 1.

Basic Stellar Data and The Resulting Parameters and Abundances.

\begin{tabular}{|c|c|c|c|c|c|c|c|c|c|c|c|c|c|c|c|}
\hline $\mathrm{HD}$ & Sp. & $T_{\text {eff }}$ & $\log g$ & $\xi^{\text {std }}$ & $v_{\mathrm{e}} \sin i$ & $\mathrm{O}$ & \multicolumn{4}{|c|}{6150 region } & $\begin{array}{l}\mathrm{C}_{53} \\
\end{array}$ & $\begin{array}{c}\mathrm{Ti} \\
80 \text { regi }\end{array}$ & $\begin{array}{l}\mathrm{Fe} \\
\text { lon }\end{array}$ & $\begin{array}{c}\mathrm{O} \\
7775\end{array}$ & $\begin{array}{c}\mathrm{Fe} \\
\text { region }\end{array}$ \\
\hline 130109 & $\mathrm{~A} 0 \mathrm{~V}$ & 9683 & 3.68 & 2.4 & 290 & -0.45 & +0.66 & & -0.82 & & . & & $\ldots$ & -0.06 & $\ldots$ \\
\hline 028024 & $\mathrm{~A} 8 \mathrm{Vn}$ & 7107 & 3.20 & 3.3 & 250 & +0.13 & +0.05 & +0.04 & +0.03 & -0.29 & $\ldots$ & -0.21 & -0.71 & +0.15 & $\ldots$ \\
\hline 106591 & A3V & 8629 & 3.85 & 3.7 & 221 & -0.36 & $\ldots$ & -0.19 & -0.51 & $\ldots$ & +0.04 & -1.18 & -0.15 & -0.48 & $\ldots$ \\
\hline 141003 & A3V & 8580 & 3.56 & 3.7 & 220 & -0.10 & . & +0.80 & +0.03 & . & -0.12 & -0.51 & -0.14 & -0.31 & $\ldots$ \\
\hline 027946 & A7V & 7401 & 3.84 & 3.7 & 193 & +0.12 & +0.20 & +0.24 & +0.10 & -0.25 & -0.47 & +0.22 & -0.43 & +0.16 & $\cdots$ \\
\hline 097603 & $\mathrm{~A} 4 \mathrm{~V}$ & 8180 & 3.90 & 4.0 & 191 & -0.01 & . & +0 . & +0.00 & -0.51 & -0.07 & -0.37 & -0.30 & -0.28 & $\cdots$ \\
\hline 059037 & $\mathrm{~A} 4 \mathrm{~V}$ & 8238 & 3.99 & 4.0 & 185 & +0.01 & .. & +0 . & +0.01 & -0.53 & -0.28 & -0.02 & -0.32 & -0.21 & $\ldots$ \\
\hline 102124 & $\mathrm{~A} 4 \mathrm{~V}$ & 8026 & 4.09 & 4.0 & 185 & -0.06 & +0.37 & +0.40 & +0.08 & -0.22 & -0.26 & -0.23 & -0.40 & -0.25 & -0.23 \\
\hline 080081 & A3V & 9014 & 3.82 & 3.3 & 179 & -0.27 & . & +0.35 & -0.34 & $\ldots$ & -0.02 & 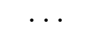 & -0.01 & -0.36 & $\ldots$ \\
\hline 103287 & A0Ve & 9202 & 3.79 & 3.0 & 164 & -0.18 & .. & -0.36 & -0.26 & $\ldots$ & -0.38 & $\cdots$ & -0.25 & -0.40 & $\ldots$ \\
\hline 056537 & A3V & 8458 & 3.90 & 3.8 & 162 & +0.01 & . & +0.41 & -0.03 & -0.40 & -0.10 & -0.08 & -0.16 & -0.23 & +0.42 \\
\hline 076644 & A7V & 7934 & 4.22 & 4.0 & 139 & +0.03 & -0.30 & +0.06 & -0.21 & -0.50 & -0.26 & +0.03 & -0.30 & -0.27 & -0.43 \\
\hline 029488 & $\mathrm{~A} 5 \mathrm{Vn}$ & 7990 & 3.82 & 4.0 & 137 & +0.12 & -0.16 & -0 . & -0.12 & -0.60 & -0.18 & -0.03 & -0.32 & -0.15 & -0.08 \\
\hline 099211 & A7Vn: & 7722 & 3.95 & 3.9 & 128 & -0.22 & -0.21 & -0 & -0.21 & -0.16 & -0.36 & -0.16 & -0.46 & -0.37 & -0.16 \\
\hline 031295 & $\mathrm{~A} 0 \mathrm{~V}$ & 8993 & 4.11 & 3 & 123 & -0.33 & . & -0.70 & -0.56 & $\cdots$ & -0.29 & $\ldots$ & -0.71 & -0.20 & $\ldots$ \\
\hline 127762 & A7III & 7663 & 3.59 & 3.9 & 123 & -0.12 & -0.25 & -0 & -0.24 & +0.07 & -0.26 & -0.05 & -0.36 & -0.25 & -0.24 \\
\hline 139006 & $\mathrm{~A} 0 \mathrm{~V}$ & 9573 & 3.87 & 2.5 & 121 & -0.17 & & +0 . & -0.15 & -0.80 & -0.07 & -0 . & -0.13 & -0.54 & \\
\hline 028527 & A6IV & 8039 & 3.99 & 4.0 & 120 & +0.15 & +0.0 & -0.0 & -0.06 & -0.54 & -0.13 & +0.11 & -0.26 & -0.03 & -0.36 \\
\hline 032301 & A7V & 7937 & 3.74 & 4.0 & 120 & +0.12 & -0.0 & -0. & -0.14 & -0.56 & -0.19 & +0.01 & -0.25 & -0.08 & -0.05 \\
\hline 102647 & A3V & 8643 & 4.17 & 3.7 & 120 & -0. & -0.4 & +0.0 & -0.12 & -0.16 & -0.24 & -0.20 & -0.11 & -0.28 & $\ldots$ \\
\hline 028910 & A $8 \mathrm{~V}$ & 7520 & 3.97 & 3.8 & 119 & -0.10 & -0.48 & -0.42 & -0.22 & -0.22 & -0.53 & -0.09 & -0.32 & -0.12 & +0.63 \\
\hline 028355 & A7V & 7809 & 3.98 & 4.0 & 91 & -0.07 & +0.11 & -0 . & +0.12 & +0.42 & -0.50 & -0.08 & -0.19 & -0.27 & +0.00 \\
\hline 027934 & A7IV-V & 8159 & 3.84 & 4.0 & 87 & +0 & +0.14 & -0. & +0.01 & -0 & -0.13 & +0. & -0.22 & -0.12 & -0.07 \\
\hline 074198 & $1 \mathrm{IV}$ & 9381 & 4.11 & 2.8 & 87 & -0 & -0 . & -0 & +0 & +0 . & -0.39 & -0 . & +0.24 & -0.25 & +0.26 \\
\hline 033641 & $4 \mathrm{~m}$ & 7961 & 4.21 & 4.0 & 86 & -0.30 & -0.18 & -0 & +0 & +0 . & -0.64 & +0.01 & -0.11 & -0.34 & -0.10 \\
\hline 028226 & A & 7361 & 4.01 & 3 & 85 & -0 . & +0.07 & -0 & +0 & +0 . & -0.25 & -0 . & -0.01 & -0.12 & +0.08 \\
\hline 029388 & $6 \mathrm{~V}$ & 8194 & 3.88 & 4.8 & 85 & +0 . & +0.08 & -0 . & -0.05 & -0.36 & -0.17 & +0.03 & -0.25 & -0.10 & -0.03 \\
\hline 025490 & $\mathrm{~A} 0.5 \mathrm{Va}$ & 9077 & 3.93 & 3. & 80 & -0.50 & . & -0.29 & -0.10 & +0.11 & -0.50 & -0.45 & -0.12 & -0.29 & +0.70 \\
\hline 028319 & A7III & 7789 & 3.68 & 4.0 & 71 & -0.01 & +0.0 & -0.20 & -0.14 & -0.41 & -0.23 & +0.02 & -0.41 & -0.25 & -0.18 \\
\hline 095382 & A5III & 8017 & 3.95 & 4. & 7( & -0.03 & -0.15 & -0.11 & -0.15 & -0.06 & -0.27 & -0.05 & -0.30 & -0.01 & +0.32 \\
\hline 116656 & $\mathrm{~A} 2 \mathrm{~V}$ & 9317 & 4.10 & 2. & 62 & -0.48 & -0.13 & -0 . & +0.26 & +0.73 & -0.96 & +0.20 & +0.10 & . & $\cdots$ \\
\hline 130841 & $\mathrm{~A} 5 \mathrm{~m}^{*}$ & 8079 & 3.96 & 4.0 & 60 & -0 . & -1.29 & -1 . & -0.39 & -0.24 & -1.59 & -0.35 & -0.45 & -0.87 & +0.15 \\
\hline 029479 & $4 \mathrm{~m}$ & 8406 & 4.14 & 3.9 & 58 & -0 & +0.13 & -0 & +0.29 & +1.08 & -0.44 & +0.18 & +0.14 & -0.12 & +0.31 \\
\hline 089021 & A2IV & 8861 & 3.61 & 3.5 & 52 & -0.25 & -0.06 & -0.25 & +0.06 & +0.53 & -0.57 & -0.08 & +0.02 & -0.30 & +0.23 \\
\hline 027819 & A7V & 8047 & 3.95 & 4.0 & 47 & -0.09 & -0.05 & +0.06 & -0.08 & -0.13 & -0.12 & -0.03 & -0.21 & -0.11 & -0.01 \\
\hline 043378 & Vs & 9210 & 4.09 & 3.0 & 46 & -0 & -0.47 & -0 & -0.17 & -0.17 & -0.29 & -0.05 & -0.21 & -0.18 & +0.18 \\
\hline 095418 & V & 9489 & 3.85 & 2.7 & 46 & -0 & -0 & -0 & +( & +0 . & -0.66 & -0.01 & +0.17 & -0.28 & +0.78 \\
\hline 107 & IV & 8665 & 4. & 3. & 38 & -0 & -0.26 & -0 & -0 & +0 . & -0.36 & +0 . & -0.13 & -0.21 & -0.03 \\
\hline 141795 & $\mathrm{~A} 2 \mathrm{~m}$ & 367 & 4.24 & 3. & 34 & -0.68 & -0.09 & -0.67 & +0.18 & +1.15 & -0.98 & -0.15 & +0.07 & -0.60 & +0.16 \\
\hline 028546 & $\Delta$ & 7640 & 4.17 & 3.9 & 28 & -0.3 & -0.03 & -0.40 & +0.12 & +0.83 & -0.46 & -0.07 & -0.07 & -0.33 & +0.11 \\
\hline 095608 & A & 972 & 4.20 & 3.3 & 18 & -0 & -0.05 & -0.86 & +0.27 & +0.97 & -1.12 & -0.01 & +0.16 & -0.58 & +0.37 \\
\hline 048915 & $A$ & 938 & 4.31 & 2.1 & 17 & -0 . & -0.21 & -0.46 & +0.40 & +1.20 & -0.91 & +0.15 & +0.41 & -0.31 & +0.49 \\
\hline 072037 & $\mathrm{~m}$ & 918 & .16 & 4.0 & 12 & -1 & +0.00 & -1 & +0.12 & +0 & -1.47 & -0.11 & -0.03 & -0.95 & +0.13 \\
\hline 027962 & A2IV & 8923 & 3.94 & 3.4 & 11 & -0.27 & +0.03 & -0.24 & +0.23 & +0.73 & -0.48 & +0.10 & +0.15 & -0.32 & +0.41 \\
\hline 047105 & A0IV & 9115 & 3.49 & 3.2 & 11 & -0.05 & -0.12 & -0.03 & -0.05 & +0.19 & -0.28 & -0.02 & -0.07 & -0.41 & +0.26 \\
\hline 040932 & $\mathrm{~A} 2 \mathrm{~V}$ & 8005 & 3.93 & 4.0 & 10 & -0.55 & -0.44 & -0.77 & -0.20 & +0.23 & -0.66 & -0.37 & -0.45 & -0.25 & -0.21 \\
\hline
\end{tabular}

In columns 1 through 5 are given the HD number, spectral type (from SIMBAD database), effective temperature (in $\mathrm{K}$ ), logarithmic surface gravity (in $\mathrm{cm} \mathrm{s}^{-2}$ ), and microturbulent velocity (in $\mathrm{km} \mathrm{s}^{-1}$ ). Columns 6 through 11 show the results determined from 6150 region fitting: the projected rotational velocity $\left(\right.$ in $\left.\mathrm{km} \mathrm{s} \mathrm{s}^{-1}\right),[\mathrm{O} / \mathrm{H}]$, $[\mathrm{Si} / \mathrm{H}],[\mathrm{Ca} / \mathrm{H}],[\mathrm{Fe} / \mathrm{H}]$, and $[\mathrm{Ba} / \mathrm{H}]$. Similarly, the abundance results from the 5380 region fitting $([\mathrm{C} / \mathrm{H}],[\mathrm{Ti} / \mathrm{H}]$, and $[\mathrm{Fe} / \mathrm{H}])$ and the 7775 region fitting $([\mathrm{O} / \mathrm{H}]$ and $[\mathrm{Fe} / \mathrm{H}])$ are given in columns $12-14$ and 15-16, respectively. All abundance results $([\mathrm{X} / \mathrm{H}])$ are the differential values relative to Procyon. The 46 stars are arranged in the descending order of $v_{\mathrm{e}} \sin i$, which are divided into three groups: rapidly-rotating $\operatorname{stars}\left(v_{\mathrm{e}} \sin i>100 \mathrm{~km} \mathrm{~s} \mathrm{~s}^{-1}\right)$, moderately-rotating stars $\left(50 \mathrm{~km} \mathrm{~s}^{-1}<v_{\mathrm{e}} \sin i<100 \mathrm{~km} \mathrm{~s}^{-1}\right)$, and slowly-rotating stars $\left(v_{\mathrm{e}} \sin i<50 \mathrm{~km} \mathrm{~s}{ }^{-1}\right)$.

* More exactly, SIMBAD gives "kA2hA5mA4Iv-v" for the spectral type of this star. 
from the colors of Strömgren's uvby $\beta$ photometric system with the help of the uvbybetanew ${ }^{\S}$ program (Napiwotzki, Schönberner, \& Wenske 1993), which is an updated/combined version of Moon's (1985) UVBYBETA (for dereddening) and TEFFLOGG (for determining $T_{\text {eff }}$ and $\log g$ ) codes while based on Kurucz's (1993) ATLAS9 models. The observed color data $\left(b-y, c_{1}, m_{1}\right.$, $\beta_{1}$ ) of each star were taken from the extensive compilation of Hauck \& Mermilliod (1980) via the SIMBAD database. The resulting values of $T_{\text {eff }}$ and $\log g$ are given in Table 1.

Then, the model atmosphere for each star was constructed by two-dimensionally interpolating Kurucz's (1993) ATLAS9 model grid in terms of $T_{\text {eff }}$ and $\log g$, where we exclusively applied the solar-metallicity models as was done in Takeda \& Sadakane (1997) or Takeda et al. (1999).

\section{ANALYSIS}

\section{(a) Method and Selected Regions}

As a numerical tool for extracting information from the spectra, we adopted the multi-parameter fitting technique developed by Takeda (1995), which can simultaneously determine various parameters affecting the spectra; e.g., abundances of elements showing lines of appreciable contributions, the projected rotational velocity, or the microturbulent velocity.

In the present study, we decided to concentrate on three wavelength regions to be analyzed: (1) 6140 6170 A region (hereinafter called "6150 region") including lines of $\mathrm{O}, \mathrm{Si}, \mathrm{Ca}, \mathrm{Fe}$, and $\mathrm{Ba}$; (2) 5375-5390 $\AA$ region ("5380 region") including lines of $\mathrm{C}, \mathrm{Ti}$, and $\mathrm{Fe}$; (3) 7765-7785 ^ region ("7775 region") including lines of $\mathrm{O}$ and $\mathrm{Fe}$.

\section{(b) Microturbulence}

One of the important key parameters is the microturbulence $(\xi)$, the choice of which can be critical in abundance determinations from strong line features. Although our method of analysis provides us with a possibility of establishing this parameter as demonstrated by Takeda (1995), whether it works successful or not depends upon situations (i.e., not always possible; especially, its difficulty grows as the rotation becomes higher). Besides, we found from experiences that solutions can sometimes converge at inappropriate (or erroneous) $\xi$ values for the cases of rapid rotators or insufficient data quality.

Accordingly, on the supposition that $\xi$ is a function of $T_{\text {eff }}$, we decided to find an appropriate analytical formula by combining the solutions of $\xi$ for the successfully determined cases. For this purpose, special preparatory multi-parameter fitting analyses "including $\xi$ as a variable" (in addition to the elemental abundances and

\footnotetext{
$\S$ Available at http://www.astro.le.ac.uk/ ${ }^{\sim}$ rn38/uvbybeta.html.
}

TABLE 2.

ATOMiC DATA OF IMPORTANT LINES.*

\begin{tabular}{crrr}
\hline \hline SPECIES & $\lambda(\AA)$ & $\chi(\mathrm{EV})$ & \multicolumn{1}{c}{$\log g f$} \\
\hline BA II & 6142.928 & 0.552 & -0.992 \\
SI I & 6143.125 & 5.964 & -2.790 \\
SI I & 6145.016 & 5.616 & -0.820 \\
FE II & 6147.741 & 3.889 & -2.721 \\
FE I & 6149.258 & 3.889 & -2.724 \\
FE I & 6151.617 & 2.176 & -3.299 \\
SI I & 6155.134 & 5.619 & -0.400 \\
O I & 6155.961 & 10.740 & -1.401 \\
O I & 6155.971 & 10.740 & -1.051 \\
O I & 6155.989 & 10.740 & -1.161 \\
O I & 6156.737 & 10.740 & -1.521 \\
O I & 6156.755 & 10.740 & -0.931 \\
O I & 6156.778 & 10.740 & -0.731 \\
O I & 6158.149 & 10.741 & -1.891 \\
O I & 6158.172 & 10.741 & -1.031 \\
O I & 6158.187 & 10.741 & -0.441 \\
CA I & 6161.297 & 2.523 & -1.020 \\
CA I & 6162.173 & 1.899 & +0.100 \\
CA I & 6163.755 & 2.521 & -1.020 \\
FE I & 6165.361 & 4.143 & -1.550 \\
CA I & 6166.439 & 2.521 & -0.900 \\
\hline FE I & 5379.574 & 3.695 & -1.480 \\
C I & 5380.224 & 8.850 & -2.030 \\
C I & 5380.265 & 8.850 & -2.820 \\
C I & 5380.265 & 8.850 & -2.820 \\
TI II & 5381.015 & 1.566 & -2.080 \\
FE I & 5383.369 & 4.312 & +0.500 \\
FE I & 5386.335 & 4.154 & -1.770 \\
FE I & 5386.959 & 3.642 & -2.624 \\
FE II & 5387.063 & 10.521 & +0.518 \\
FE I & 5387.488 & 4.143 & -2.140 \\
\hline O I & 7771.944 & 9.146 & +0.324 \\
O I & 7774.166 & 9.146 & +0.174 \\
O I & 7775.388 & 9.146 & -0.046 \\
FE II & 7780.354 & 9.761 & -0.547 \\
FE I & 7780.552 & 4.473 & -0.066 \\
\hline & & & \\
\hline
\end{tabular}

* All data were taken from the compilation of Kurucz $\&$ Bell (1995), except for the $g f$ value of the Fe I line at $7780.552 \AA$, for which we used Kurucz \& Peytremann's (1975) value in accordance with Takeda \& Sadakane (1997).

the rotational velocity focused in the standard analysis described in Sect. IV-c) were first carried out for the 6150 region and the 7775 region with an intention to derive the $\xi^{\text {fit }}$ values (denoted as $\xi_{6150}^{\text {fit }}$ and $\xi_{7775}^{\text {fit }}$, respectively). It turned out that $\xi_{6150}^{\mathrm{fit}}$ and $\xi_{7775}^{\mathrm{fit}}$ could be determined for 33 and 13 stars, respectively, out of 46 program stars.

The correlation of $\xi_{6150}^{\mathrm{fit}}$ and $\xi_{7775}^{\mathrm{fit}}$ for 13 stars in common is displayed in Figure 2a, where we do not recognize any systematic discordance between these two, 
though the scatter is rather large (the average difference is $\sim 0.1 \mathrm{~km} \mathrm{~s}^{-1}$ with the standard deviation of $\left.\sim 1 \mathrm{~km} \mathrm{~s}^{-1}\right)$. Figure $2 \mathrm{~b}$ shows these $\xi_{6150}^{\mathrm{fit}}$ and $\xi_{7775}^{\mathrm{fit}}$ values plotted against $T_{\text {eff }}$, where the $\xi$ results derived for $\mathrm{F}-\mathrm{G}-\mathrm{K}$ dwarfs/subgiants are also overplotted for comparison. We can see from this figure that, as $T_{\text {eff }}$ becomes higher, $\xi^{\text {fit }}$ increases from $\sim 1 \mathrm{~km} \mathrm{~s}^{-1}$ (at $T_{\text {eff }} \sim 6000 \mathrm{~K}$ ), to its nearly maximum value of $\sim 4( \pm 2) \mathrm{km} \mathrm{s}^{-1}$ (at $\left.T_{\text {eff }} \sim 8000 \mathrm{~K}\right)$ though with a considerably large scatter, followed by a decreasing tendency toward higher $T_{\text {eff }}$ of $\sim 10000 \mathrm{~K}$ (where we know $\xi$ is typically $\sim 1-2 \mathrm{~km} \mathrm{~s}^{-1}$; cf. Sadakane 1990). Hence, we adopt an analytical formula for the standard microturbulence $\left(\xi^{\text {std }}\right)$

$$
\xi^{\text {std }}=4.0 \exp \left\{-\left[\log \left(T_{\text {eff }} / 8000\right) / A\right]^{2}\right\}
$$

(where $A \equiv[\log (10000 / 8000)] / \sqrt{\ln 2}$ ) with probable uncertainties of $\pm 30 \%$, which roughly represents (and encompasses) the observed trend as shown in Figure 2 b. Note that such $\xi^{\text {std }}$ vs. $T_{\text {eff }}$ relation we have defined is in reasonable agreement with previous results (see, e.g., Figure 1 in Coupry \& Burkhart 1992 or Figure 2 in Gebran \& Monier 2007). The $\xi$ values for each of the 46 stars evaluated by Equation (1), which we will use for abundance determinations, are given in Table 1 .

\section{(c) Solutions}

Now that the model atmosphere and the microturbulence have been assigned to each star, we can go on to evaluations of elemental abundances by way of synthetic spectrum fitting applied to three wavelength regions. All the atomic data (wavelength, excitation potential, oscillator strengths, damping constants) relevant to the analysis were taken from the extensive compilation of Kurucz \& Bell (1995), except for log $g f$ (Fe I 7780.552) (cf. the caption of Table 2). The adopted data of important lines are summarized in Table 2. The non-LTE effect was explicitly taken into account only for the O I triplet lines at 7771-5 $\AA$ (for which the nonLTE correction is known to be appreciably large and its inclusion is necessary; see, e.g., Takeda \& Sadakane 1997) based on the statistical equilibrium calculation for O I (cf. Takeda 2003); otherwise, we assumed LTE.

Applying our automatic fitting approach, we adjusted the following parameters to accomplish the best fit at each region: $A_{6150}^{\mathrm{O}}, A_{6150}^{\mathrm{Si}}, A_{6150}^{\mathrm{Ca}}, A_{6150}^{\mathrm{Fe}}, A_{6150}^{\mathrm{Ba}}$, and $v_{\mathrm{e}} \sin i_{6150}$ (for the 6150 region); $A_{5380}^{\mathrm{C}}, A_{5380}^{\mathrm{Ti}}$, $A_{5380}^{\mathrm{Fe}}$, and $v_{\mathrm{e}} \sin i_{5380}$ (for the 5380 region); and $A_{7775}^{\mathrm{O}}$, $A_{7775}^{\mathrm{Fe}}$, and $v_{\mathrm{e}} \sin i_{7775}$ (for the 7775 region).

In case that abundance solutions for some elements did not converge (especially for rapid rotators), we had to abandon their determinations and fix them at the solar abundances (i.e., abundances used in the model atmosphere) during the iteration procedure and concentrate on the remaining parameters. Figures 3 (6150 region), 4 (5380 region), and 5 (7775 region) show how the theoretical synthetic spectra corresponding to the final solutions fit the observations.

In order to demonstrate the importance (or unimportance) of the choice of microturbulence for each element, we show the abundance differences between the two cases of $\xi^{\text {fit }}$ and $\xi^{\text {std }}$ in Figures 6a (6150 region) and $b$ (7775 region) by using the abundance results obtained as by-products from the $\xi^{\text {fit }}$-determinination mentioned in the previous Sect. IV-b. It can be seen from these figures that the $A$ values are not very sensitive to the choice of $\xi$, except that only $A^{\mathrm{Ba}}$ is considerably $\xi$-dependent because the Ba II line at $6142.9 \AA$ (on which $A^{\mathrm{Ba}}$ essentially relies) is strongly saturated (cf. Figure 3). In the remainder of this paper, we exclusively refer to the abundances derived by using $\xi^{\text {std }}$ as the standard abundances to be discussed.

We also estimated the uncertainties in $A^{\mathrm{X}}$ by repeating the analysis while perturbing the standard values of the atmospheric parameters $\left(T_{\mathrm{eff}}^{\mathrm{std}}, \log g^{\mathrm{std}}\right.$, $\left.\xi^{\text {std }}\right)$ interchangeably by $\pm 300 \mathrm{~K}, \pm 0.3 \mathrm{dex}$, and $\pm 0.3 \xi^{\text {std }} \mathrm{km} \mathrm{s}^{-1}$. Then, the root-sum-square of the resulting abundance changes $\left(\Delta_{T}, \Delta_{g}, \Delta_{\xi}\right)$ may be regarded as the error involved in $A^{\mathrm{X}}$; i.e., $\Delta A^{\mathrm{X}} \equiv$ $\left(\Delta_{T}^{2}+\Delta_{g}^{2}+\Delta_{\xi}^{2}\right)^{1 / 2}$

In discussing abundance peculiarities, it is useful to represent the results of elemental abundances in terms of the differences relative to the fiducial values. Unfortunately, the Sun is not suitable for this purpose since its spectrum appearance is considerably different from that of A-type stars. Accordingly, we adopted Procyon (F5 IV-V) as the reference star of abundance standard, considering that it has parameters not very different from those of A stars and its chemical composition is known to be nearly the same as that of the Sun (cf., e.g., Kato \& Sadakane 1982, Steffen 1985, or Edvardsson et al. 1993; see also Figure 3 in Varenne \& Monier 1999). Regarding the spectra of Procyon, we used Takeda et al.'s (2005a) OAO spectrum database for the 6160 and 5380 regions, while Allende Prieto et al.'s (2004) publicdomain spectrum was invoked for the 7775 region. Adopting Takeda et al.'s (2005b) results for the atmospheric parameters $\left(T_{\text {eff }}=6612 \mathrm{~K}, \log g=4.00\right.$, and $\xi=2.0 \mathrm{~km} \mathrm{~s}^{-1}$ ), we derived the elemental abundances of Procyon," from which $[\mathrm{X} / \mathrm{H}]$ values (star-Procyon differential abundances) were computed as $[\mathrm{X} / \mathrm{H}]_{\text {region }}$

\footnotetext{
ฯWe consider that typical uncertainties in $T_{\text {eff }}$ and $\log g$ determinations for A-type stars are roughly on the order of $\sim 300 \mathrm{~K}$ and $\sim 0.3$ dex, respectively, which we inferred from dispersions in the literature values of stellar atmospheric parameters (e.g., Cayrel de Strobel, Soubiran, \& Ralite 2001 or Sadakane \& Okyudo 1989). Besides, as mentioned in Sect. IV-b, the ambiguity in $\xi$ is estimated to be $\pm 30 \%$.

$\|$ The resulting abundances of Procyon (in the usual normalization of $\left.A^{\mathrm{H}}=12.00\right)$ are as follows: $A_{6150}^{\mathrm{O}}=8.87, A_{6150}^{\mathrm{Si}}=7.14$, $A_{6150}^{\mathrm{Ca}}=6.19, A_{6150}^{\mathrm{Fe}}=7.49$, and $A_{6150}^{\mathrm{Ba}}=2.33$ (for the 6150 region); $A_{5380}^{\mathrm{C}}=8.75, A_{5380}^{\mathrm{Ti}}=5.15$, and $A_{5380}^{\mathrm{Fe}}=7.55$ (for the 5380 region); and $A_{7775}^{\mathrm{O}}=8.90$ and $A_{7775}^{\mathrm{Fe}}=7.40$ (for the 7775 region).
} 

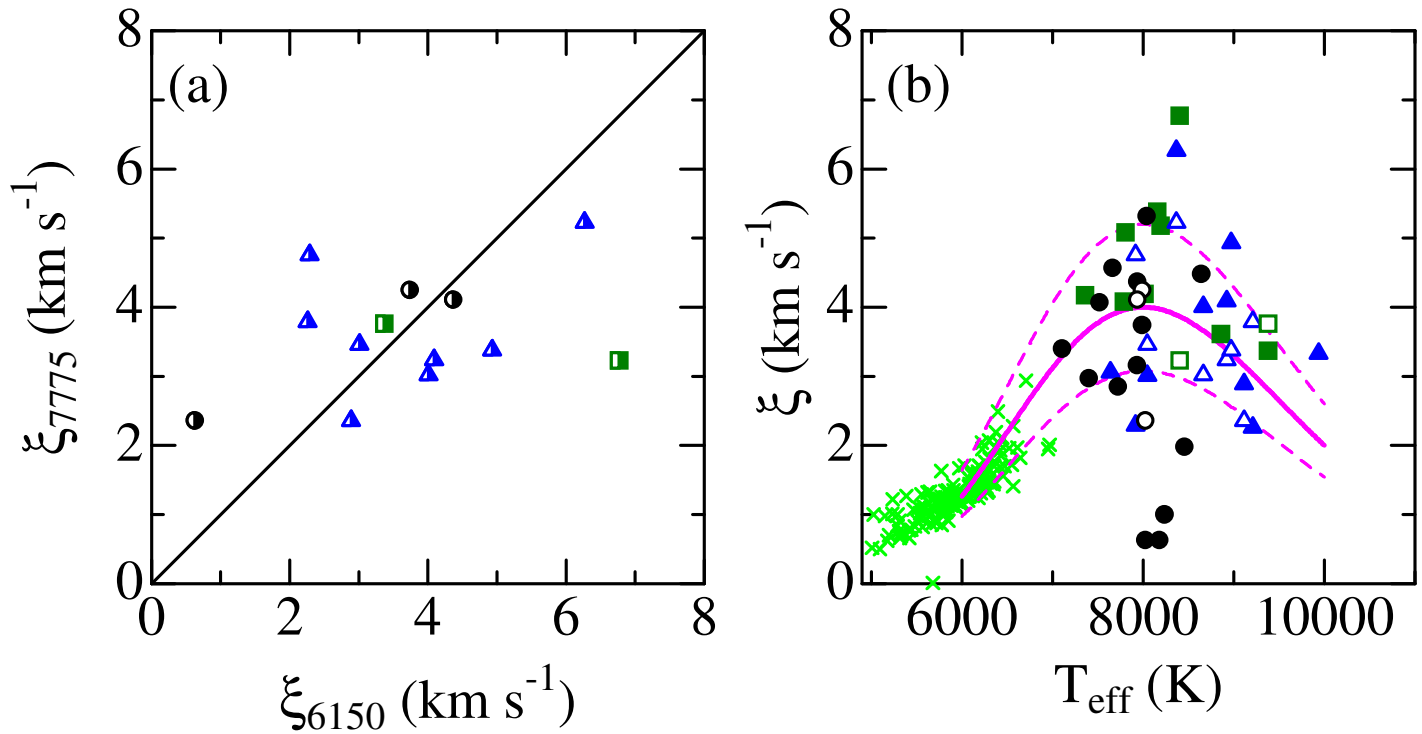

Fig. 2. - (a) Comparison of the microturbulence derived from the 7775 region $\left(\xi_{7775}\right)$ with that from the 6150 region $\left(\xi_{6150}\right)$. (b) Microturbulences plotted against the effective temperature, where $\xi_{6150}$ and $\xi_{7775}$ are denoted by filled and open symbols, respectively. The results for $\mathrm{F}-\mathrm{G}-\mathrm{K}$ dwarfs taken from Takeda et al. (2005b) are also shown by crosses for comparison. In both panels, three groups of projected rotational velocity are discerned by the symbol shape: Triangles... slow rotators $\left(0 \mathrm{~km} \mathrm{~s}^{-1}<v_{\mathrm{e}} \sin i<50 \mathrm{~km} \mathrm{~s}^{-1}\right)$, squares $\cdots$ moderate rotators $\left(50 \mathrm{~km} \mathrm{~s}^{-1}<v_{\mathrm{e}} \sin i<100 \mathrm{~km} \mathrm{~s}{ }^{-1}\right)$, and circles $\cdots$ rapid rotators $\left(100 \mathrm{~km} \mathrm{~s}^{-1}<v_{\mathrm{e}} \sin i\right)$. The adopted $\xi$ vs. $T_{\text {eff }}$ relation [cf. Equation (1)] is depicted by solid line, while its reasonable upper and lower limits (perturbations by $\pm 30 \%$ as possible range of errors) are shown by two dashed lines.

$\equiv A_{\text {region }}^{\mathrm{X}}(\mathrm{star})-A_{\text {region }}^{\mathrm{X}}($ Procyon $),(\mathrm{X}$ is any of $\mathrm{C}, \mathrm{O}$, $\mathrm{Si}, \mathrm{Ca}, \mathrm{Ti}, \mathrm{Fe}, \mathrm{Ba}$; and region is any of 6150,5380 , and $7775)$. Such obtained results of $[\mathrm{X} / \mathrm{H}]$ are presented in Table 1. Comparisons of $[\mathrm{Fe} / \mathrm{H}]_{5380}$ vs. $[\mathrm{Fe} / \mathrm{H}]_{6150}$, $[\mathrm{Fe} / \mathrm{H}]_{7775}$ vs. $[\mathrm{Fe} / \mathrm{H}]_{6150}$, and $[\mathrm{O} / \mathrm{H}]_{7775}$ vs. $[\mathrm{O} / \mathrm{H}]_{6150}$ are shown in Figures $7 \mathrm{a}, \mathrm{b}$, and c, respectively. We can see from these figures that the discrepancies tend to be larger for rapid rotators, which indicates the growing difficulty in abundance determinations of broad-line stars.

From now on, in case where two or three kinds of solutions are available from different wavelength regions, we will use the result from the 6150 region (which is wider and include more lines than the other two).

The resulting rotational velocities $\left(v_{\mathrm{e}} \sin i_{6150}\right)$ are compared with the previously published results by Abt \& Morrell (1995) and Royer et al. (2002a, b) in Figure 8 , where we can recognize that our $v_{\mathrm{e}} \sin i$ solutions are in reasonable agreement with these literature values.

\section{DISCUSSION}

\section{(a) Rotation-Abundance Connection}

Figures 9a-f display the resulting $[\mathrm{X} / \mathrm{H}]$ vs. $[\mathrm{Fe} / \mathrm{H}]$ correlations (for $\mathrm{X}=\mathrm{C}, \mathrm{O}, \mathrm{Si}, \mathrm{Ca}, \mathrm{Ti}$, and $\mathrm{Ba}$ ), from which we can roughly divide these elements into three groups.

(i) Si and Ti: almost scaling in accordance with Fe.

(ii) $\mathrm{C}$, O, and $\mathrm{Ca}$ : showing an anti-correlation trend with Fe.

(iii) Ba: positive correlation with $\mathrm{Fe}$, though its range of peculiarity is much more conspicuous than that of Fe.

Besides, $[\mathrm{C} / \mathrm{H}],[\mathrm{O} / \mathrm{H}],[\mathrm{Si} / \mathrm{H}],[\mathrm{Ca} / \mathrm{H}],[\mathrm{Ti} / \mathrm{H}],[\mathrm{Ba} / \mathrm{H}]$, and $[\mathrm{Fe} / \mathrm{H}]$ are plotted against $v_{\mathrm{e}} \sin i$ in Figures 10ag. We can recognize from these figures that $[\mathrm{C} / \mathrm{H}]$, $[\mathrm{O} / \mathrm{H}],[\mathrm{Ca} / \mathrm{H}]$ and $[\mathrm{Ba} / \mathrm{H}]$ are systematically $v_{\mathrm{e}} \sin i$ dependent in the sense that the peculiarity (overabundance for $\mathrm{Ba}$, underabundance for $\mathrm{C} / \mathrm{O} / \mathrm{Ca}$ ) tends to decrease with an increase in $v_{\mathrm{e}} \sin i$. While such a convincing tendency is not apparent for the remaining elements ( $\mathrm{Si}, \mathrm{Ti}$, and $\mathrm{Fe}$ ), $[\mathrm{Fe} / \mathrm{H}]$ appears to weakly conform to this trend (i.e., decreasing tendency with $\left.v_{\mathrm{e}} \sin i\right)$.

Combining these observational fact, we may conclude as follows:

- (a) All the seven elements exhibit some kind of abundance peculiarities, which are more conspicuously seen in slow rotators $\left(v_{\mathrm{e}} \sin i \lesssim 50 \mathrm{~km} \mathrm{~s}^{-1}\right)$ and characterized by the deficiency of $\mathrm{C}, \mathrm{O}$, and $\mathrm{Ca}$ and the enrichment of $\mathrm{Si}, \mathrm{Fe}$, and (especially) Ba. 




Fig. 3.- Synthetic spectrum fitting at the 6150 region (6140-6170 A) for determining the projected rotational velocity $\left(v_{\mathrm{e}} \sin i\right)$ and the abundances of $\mathrm{O}, \mathrm{Si}, \mathrm{Ca}, \mathrm{Fe}$, and $\mathrm{Ba}$. The best-fit theoretical spectra are shown by solid lines, while the observed data are plotted by symbols. Left panel $\cdots$ rapid rotators $\left(100 \mathrm{~km} \mathrm{~s}^{-1}<v_{\mathrm{e}} \sin i\right)$, middle panel $\cdots$ moderate rotators $\left(50 \mathrm{~km} \mathrm{~s}^{-1}<v_{\mathrm{e}} \sin i<100 \mathrm{~km} \mathrm{~s}^{-1}\right)$, and right panel $\cdots$ slow rotators $\left(0 \mathrm{~km} \mathrm{~s}^{-1}<v_{\mathrm{e}} \sin i<50 \mathrm{~km} \mathrm{~s}{ }^{-1}\right)$. In each panel, the spectra are arranged in the descending order of $v_{\mathrm{e}} \sin i$, and an appropriate offset is applied to each spectrum (indicated by the HD number) relative to the adjacent one. The case of Procyon (standard star) is displayed at the bottom of the right panel. 


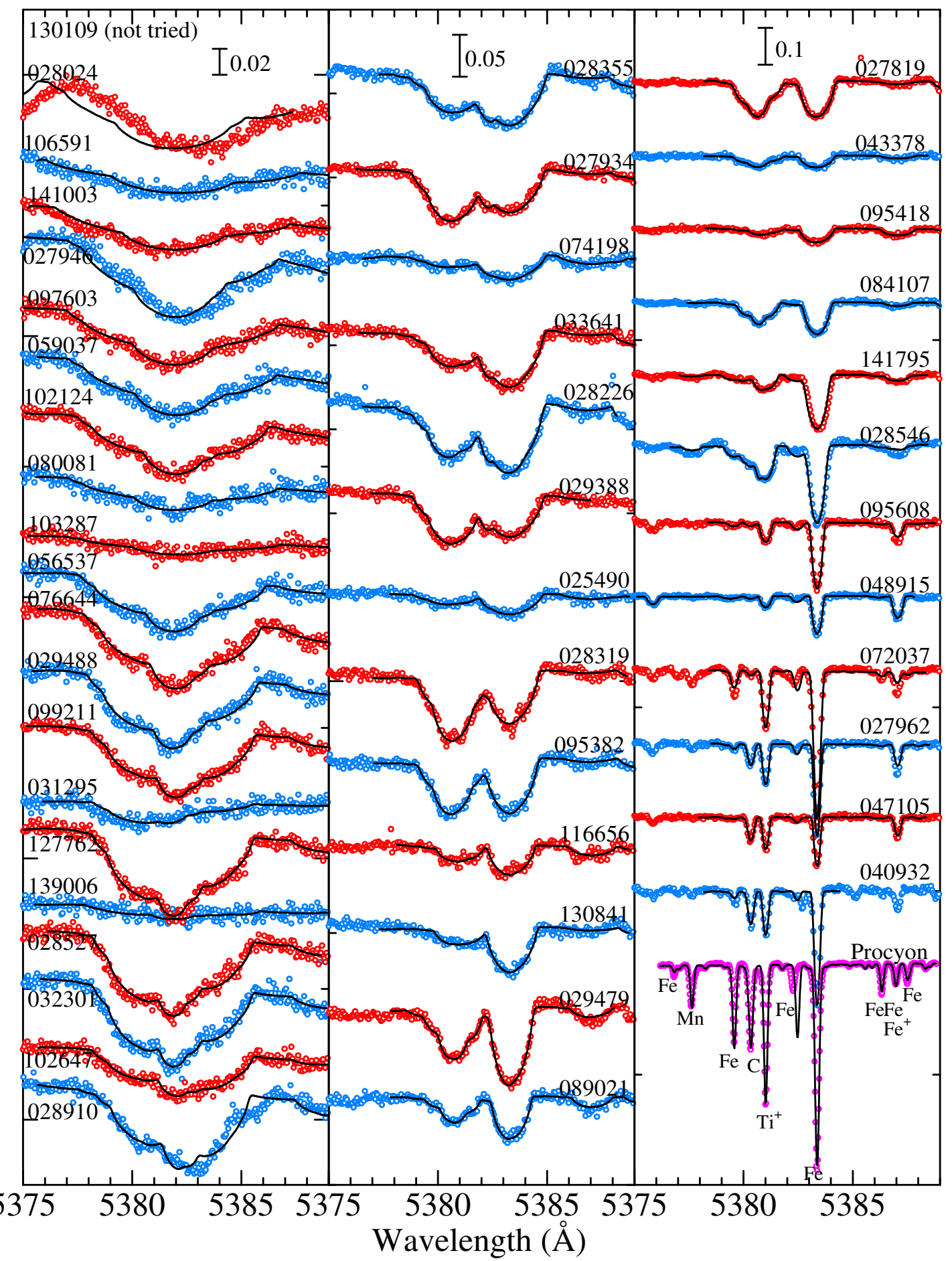

Fig. 4. - Synthetic spectrum fitting at the 5380 region $(5375-5390 \AA)$ for determining $v_{\mathrm{e}} \sin i$ and the abundances of C, $\mathrm{Ti}$, and Fe. Otherwise, the same as in Fig. 3. 


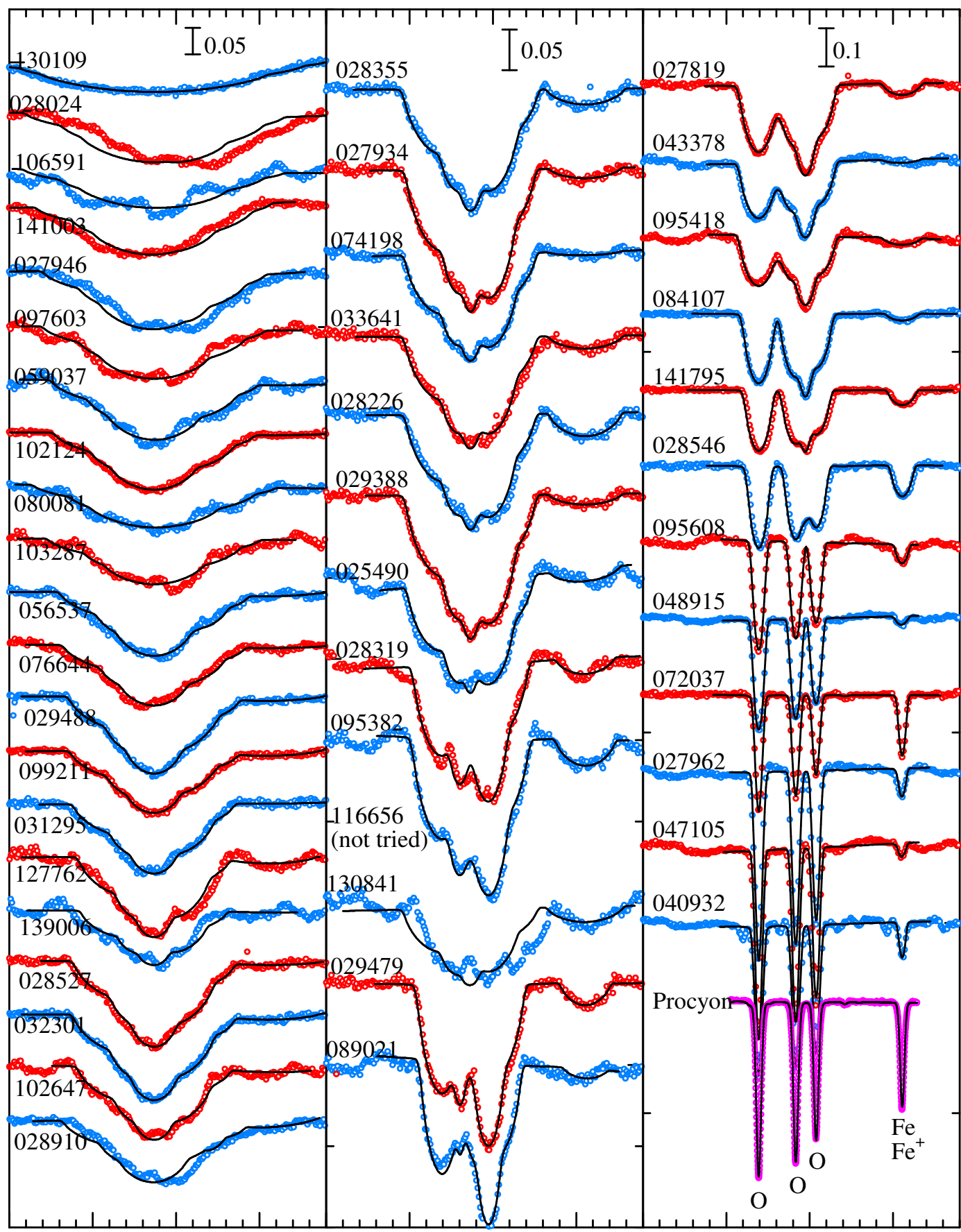

776577707775778077657770777577807765777077757780

Wavelength $(\AA)$

Fig. 5. - Synthetic spectrum fitting at the 7775 region $(7765-7785 \AA)$ for determining $v_{\mathrm{e}} \sin i$ and the abundances of $\mathrm{O}$ (with the non-LTE effect taken into account) and Fe. Otherwise, the same as in Fig. 3. 

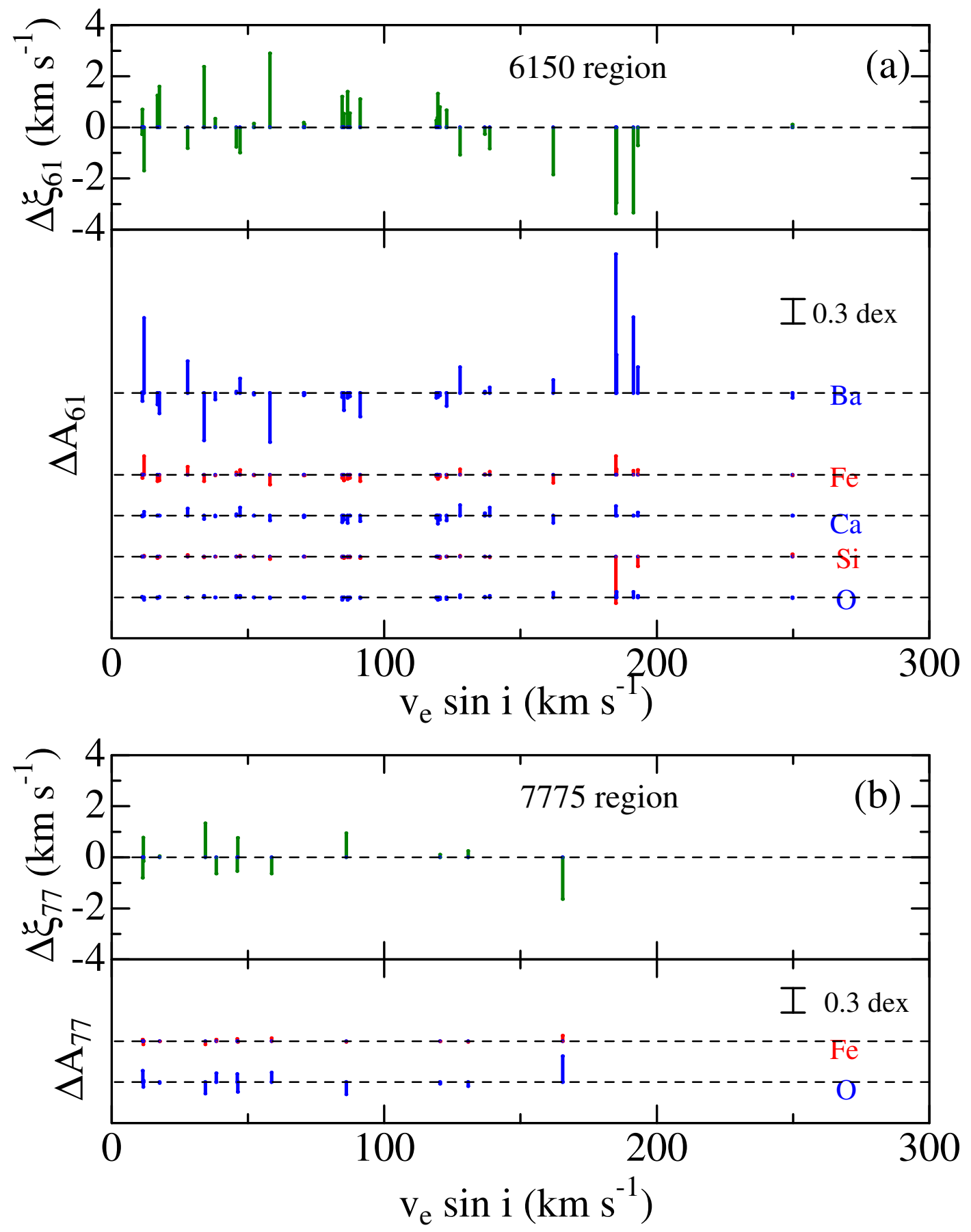

Fig. 6. - Abundance changes between the two cases of $\xi^{\text {fit }}$ and $\xi^{\text {std }}\left[\Delta A \equiv A\left(\xi^{\mathrm{fit}}\right)-A\left(\xi^{\mathrm{std}}\right)\right]$ plotted as functions of $v_{\mathrm{e}} \sin i$. (a) 6150 region $\left(33\right.$ stars) and (b) 7775 region $(13$ stars $)$. In each figure, the upper and lower panels show $\Delta \xi \equiv\left(\xi^{\text {fit }}-\xi^{\text {std }}\right)$ and $\Delta A$ (for each element), respectively. 

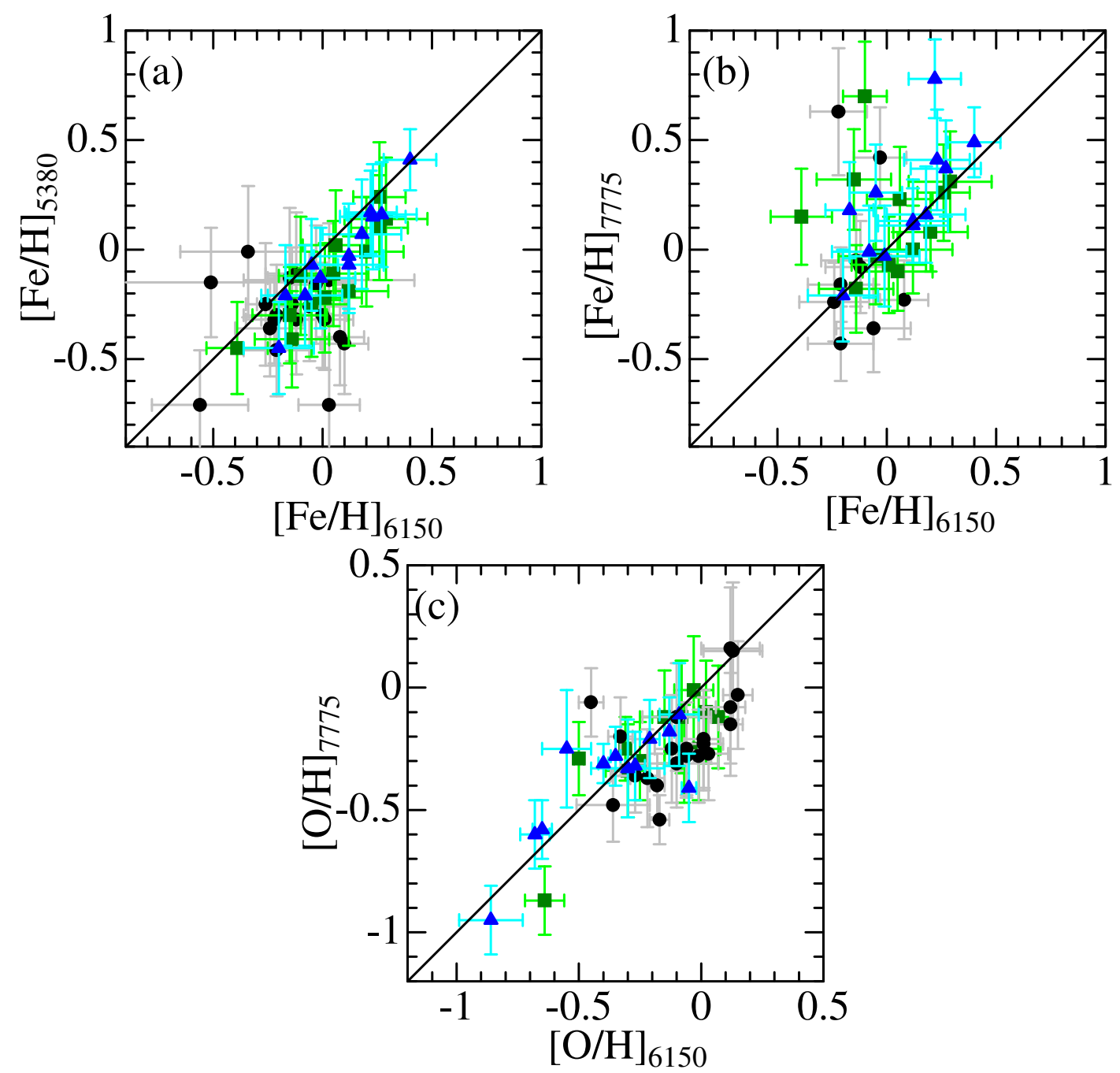

Fig. 7.- Comparisons of the abundances derived from different regions (cf. Table 1). (a) $[\mathrm{Fe} / \mathrm{H}]_{5380} \mathrm{vs.}[\mathrm{Fe} / \mathrm{H}]_{6150},(\mathrm{~b})$ $[\mathrm{Fe} / \mathrm{H}]_{7775}$ vs. $[\mathrm{Fe} / \mathrm{H}]_{6150}$, and (c) $[\mathrm{O} / \mathrm{H}]_{7775}$ vs. $[\mathrm{O} / \mathrm{H}]_{6150}$. The error bars attached in $[\mathrm{X} / \mathrm{H}]$ represent the values of $\Delta A^{\mathrm{X}}$ (see Sect. IV-c). Three groups of different $v_{\mathrm{e}} \sin i$ ranges are discriminated by the symbol shape in the same manner as in Fig. 2.

- (b) These anomalies tend to diminish progressively with an increase in $v_{\mathrm{e}} \sin i$ (at least in the range of slow/moderate rotators of $\lesssim 100 \mathrm{~km} \mathrm{~s}^{-1}$ ).

- (c) The stellar rotational velocity must thus be the most important key factor in the sense that the extent of abundance peculiarity tends to be larger as a star rotates more slowly, which is presumably because some counter-acting mechanism of diluting the built-up anomaly (most probably due to the element segregation in a stable atmosphere/envelope) takes place in rapid rotators.

We also point out these tendencies seen in Figures
9 and 10 are more or less consistent with the results of recently published papers focused on the abundance trends of A-type stars (including Am stars) for a wide range of $v_{\mathrm{e}} \sin i$ values: e.g., Lemke $(1990,1993)$ [field stars; C, Ba (elements in common with this study)], Savanov (1995a,b) [field stars; C, O, Si, Ca, Fe, Ba], Takeda \& Sadakane (1997) [Hyades and field stars; Fe, O], Gebran, Monier, \& Richard (2008) [Coma Berenices; C, O, Si, Ca, Fe, Ba], Gebran \& Monier (2008) [Pleiades; C, O, Si, Ca, Fe, Ba], and Fossati et al. (2008)[Praesepe; C, O, Si, Ca, Fe, Ba]. 



Fig. 8. - Comparison of the $v_{\mathrm{e}} \sin i$ results derived in this study (determined from the fitting in the 6150 region; cf. Table 1). with the literature values: (a) Abt \& Morrell (1995), (b) Royer et al. (2002a; open symbols; southern hemisphere) and Royer et al. (2002b; filled symbols; northern hemisphere).

\section{(b) Implication of Subsolar Compositions in Normal A Stars}

According to what we learned in Sect. V-a, we may assume that the abundance peculiarities of A-type stars (conspicuously seen slow rotators) tend to disappear for rapid rotators at $v_{\mathrm{e}} \sin i \gtrsim 100 \mathrm{~km} \mathrm{~s}^{-1}$ (cf. Figure 10). If so, we would be able to gain information of the galactic gas $\lesssim 10^{9}$ yr ago by inspecting the photospheric abundances of such rapidly-rotating A-type stars, since they are considered to retain the composition of the gas from which they formed.

From this point of view, it is interesting to note in Figure 10 that the $[\mathrm{X} / \mathrm{H}]$ values at the high $-v_{\mathrm{e}} \sin i$ range tend to be somewhat negative or "subsolar" for many elements such as $\mathrm{C}, \mathrm{O}, \mathrm{Ti}, \mathrm{Fe}$, and Ba; i.e., by several tenths dex below the solar (or Procyon) abundances on the average.

Here we recall Takeda, Sato, \& Murata's (2008) conclusion that the $[\mathrm{Fe} / \mathrm{H}]$ values (as well as those of other elements whose abundances almost scale with $\mathrm{Fe}$ ) of evolved $\mathrm{G}$ giants, many of which have mass values around $\sim 2 M_{\odot}$ like A-type dwarfs, spread in a range of $-0.8 \lesssim[\mathrm{Fe} / \mathrm{H}] \lesssim+0.2$ around an average value of $[\mathrm{Fe} / \mathrm{H}] \sim-0.3$

Considering these two observational consequences, we would conclude that the metallicities of the galactic gas $\lesssim 10^{9}$ yr ago had really a subsolar tendency (though with a rather large diversity). If this is the case, the gas metallicity of $[\mathrm{Fe} / \mathrm{H}] \sim 0\left(\sim 5 \times 10^{9}\right.$ ago when our Sun was born) must have decreased by several tenths dex with an elapse of time until $\lesssim 10^{9}$ yr ago when A dwarfs (progenitors of $\mathrm{G}$ giants) were born. Although this trend does not seem to have been taken very seriously so far ${ }^{* *}$ in spite of not a few supportive evidences ${ }^{\dagger \dagger}$ since it contradicts the conventional scenario of galactic chemical evolution (where elemental abundances are generally believed to increase with time), we tend to regard this tendency as real, which means that the gas metallicity actually decreased in an elapse of time between the formation of our Sun $\left(\sim 5 \times 10^{9}\right.$ yr ago $)$ and the formation of $\sim 2 M_{\odot}$ stars $\left(\lesssim 10^{9} \mathrm{yr}\right.$ ago). Of course, in order to make this hypothesis more convincing, a reasonable explanation has to be done why such a reduction of the gas metallicity had occurred against the intuitive chemical evolution picture of increasing metallicity. While one such interpretation might be the dilution of the metallicity caused by an substantial infall of metal-poor primordial galactic gas speculated

** Meanwhile, a completely different solution to this problem has also been proposed, arguing the necessity of downward revision of the solar abundances as a result of the application of sophisticated 3D line formation theory; (cf. Asplund et al. 2004). While this possibility may be worth considering, it can not yet be regarded as reliable in our opinion, since it causes serious discrepancies between theory and observation in the solar interior model (see, e.g., Young 2005 and the references therein). Besides, some questionable points still remain in their line-formation treatment (see also Appendix 1 in Takeda \& Honda 2005).

††Actually, the apparent subsolar tendency in the photospheric abundances of comparatively young stars has often been reported; e.g., C/N/O in early B main-sequence stars (Gies \& Lambert 1992, Kilian 1992, see also Nissen 1993); C/N/O/Si/Mg/Al in early B stars (Kilian 1994); $[\mathrm{Fe} / \mathrm{H}]$ in superficial normal late $\mathrm{B}$ and A stars (Sadakane 1990); [Fe/H] of B stars from UV spectra (Niemczura 2003); O in supergiants (Luck \& Lambert 1985; Takeda \& Takada-Hidai 1998). 

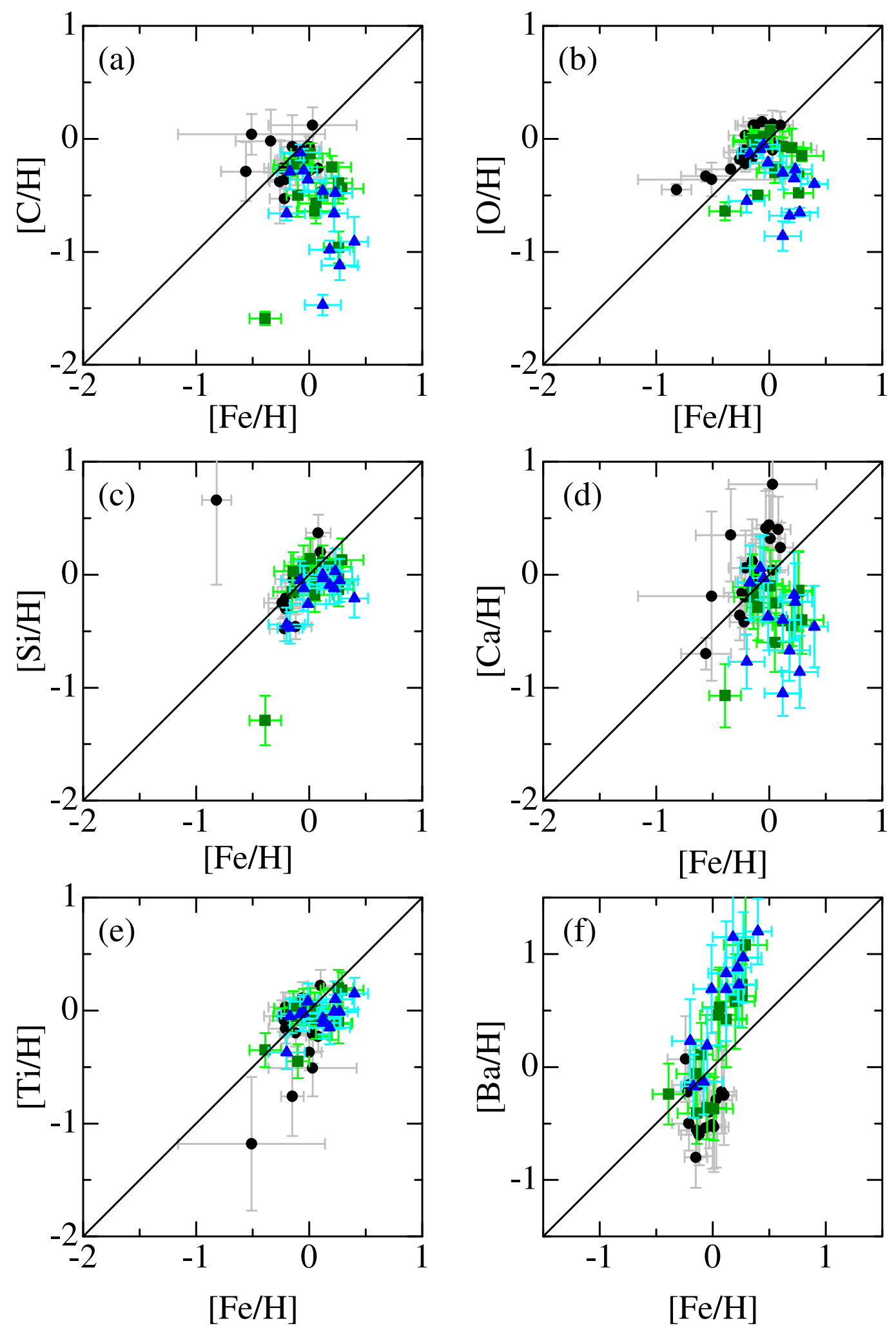

Fig. 9. - $[\mathrm{X} / \mathrm{H}]$ values plotted against $[\mathrm{Fe} / \mathrm{H}](6150$ region). (a) $[\mathrm{C} / \mathrm{H}],(\mathrm{b})[\mathrm{O} / \mathrm{H}](6150$ region), (c) $[\mathrm{Si} / \mathrm{H}],(\mathrm{d})[\mathrm{Ca} / \mathrm{H}],(\mathrm{e})$ $[\mathrm{Ti} / \mathrm{H}]$, and $(\mathrm{f})[\mathrm{Ba} / \mathrm{H}]$. The error bars attached in $[\mathrm{X} / \mathrm{H}]$ represent the values of $\Delta A^{\mathrm{X}}$ (see Sect. IV-c). See the caption of Fig. 2 for the meanings of the symbols. 

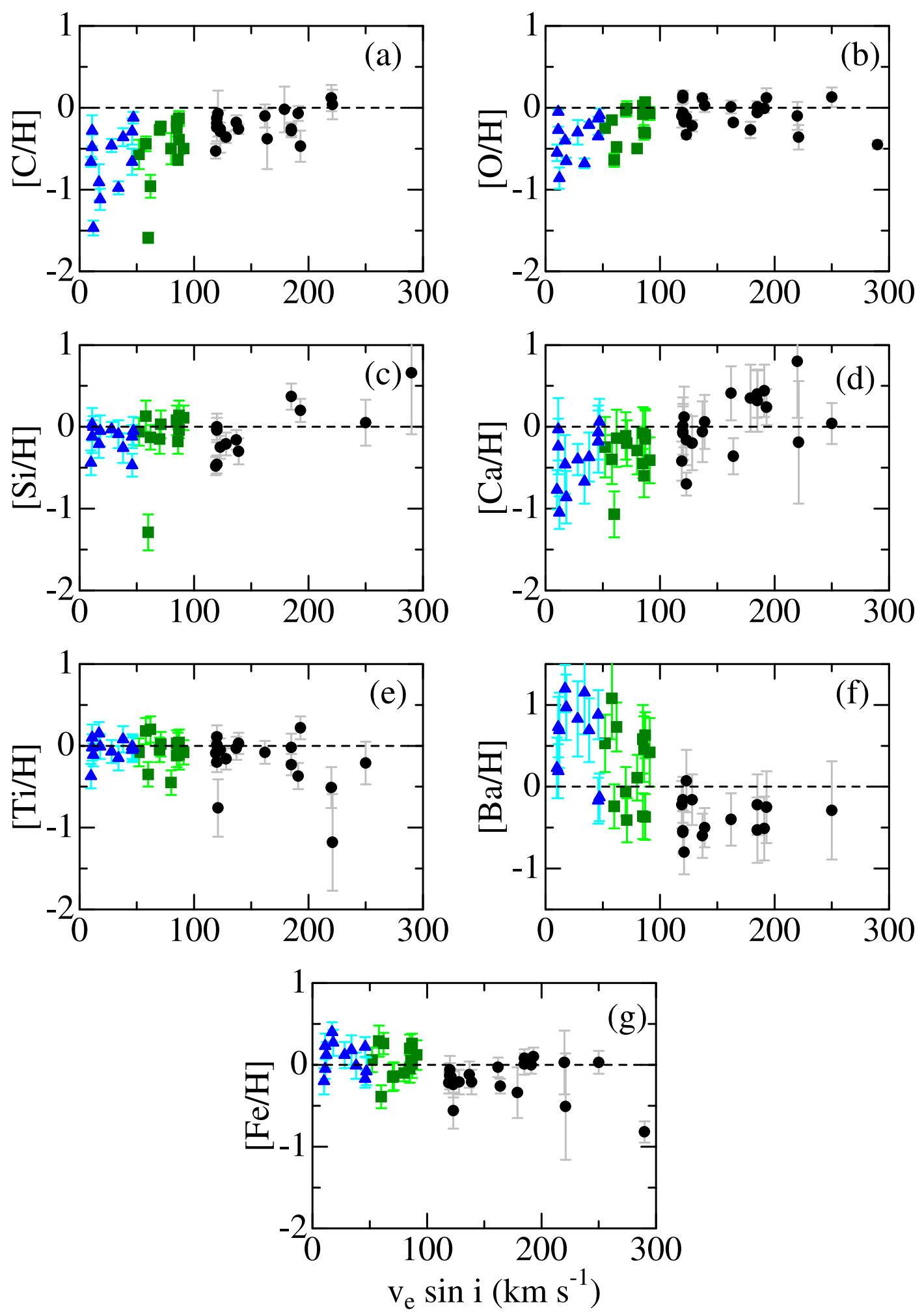

Fig. 10. - $[\mathrm{X} / \mathrm{H}]$ values plotted against $v_{\mathrm{e}} \sin i(6150$ region). (a) $[\mathrm{C} / \mathrm{H}],(\mathrm{b})[\mathrm{O} / \mathrm{H}](6150$ region), (c) $[\mathrm{Si} / \mathrm{H}],(\mathrm{d})[\mathrm{Ca} / \mathrm{H}]$, (e) $[\mathrm{Ti} / \mathrm{H}],(\mathrm{f})[\mathrm{Ba} / \mathrm{H}]$, and $(\mathrm{g})[\mathrm{Fe} / \mathrm{H}]\left(6150\right.$ region). The error bars attached in $[\mathrm{X} / \mathrm{H}]$ represent the values of $\Delta A^{\mathrm{X}}($ see Sect. IV-c). See the caption of Fig. 2 for the meanings of the symbols. 
by Takeda et al. (2008), further observations and extensive abundance analyses on a much larger number of rapidly-rotating $\mathrm{A}$ dwarfs (as well as evolved $\mathrm{G}$ giants) would be required until we can say something about it with confidence.

\section{ACKNOWLEDGEMENTS}

We express our heartful thanks to Mr. Jin-Guk Seo for his technical support during the observations.

I. Han acknowledges the financial support for this study by KICOS through Korea-Ukraine joint research grant (grant 07-179).

B.-C. Lee acknowledges the Astrophysical Research Center for the Structure and Evolution of the Cosmos (ARSEC, Sejong University) of the Korea Science and Engineering Foundation (KOSEF) through the Science Research Center (SRC) program.

\section{REFERENCES}

Abt, H. A. \& Morrell, N. I., 1995, The Relation between Rotational Velocities and Spectral Peculiarities among A-Type Stars, ApJS, 99, 135

Allende Prieto, C., Barklem, P. S., Lambert, D. L., \& Cunha, K., 2004, $\mathrm{S}^{4} \mathrm{~N}$ : A spectroscopic survey of stars in the solar neighborhood. The Nearest 15 pc, A\&A, 420, 183

Andrievsky, S. M., et al., 2002, The elemental abundance pattern of twenty lambda Bootis candidate stars, A\&A, 396, 641

Asplund, M., Grevesse, N., Sauval, A. J., Allende Prieto, C., \& Kiselman, D., 2004, Line formation in solar granulation. IV. [O I], O I and $\mathrm{OH}$ lines and the photospheric $\mathrm{O}$ abundance, A\&A, 417, 751

Cayrel de Strobel, G., Soubiran, C., \& Ralite, N., 2001, Catalogue of $[\mathrm{Fe} / \mathrm{H}]$ determinations for FGK stars: 2001 edition, A\&A, 373, 159

Coupry, M. F. \& Burkhart, C., 1992, The A and AmFm stars. II - Equivalent widths for the Li region, microturbulence, and comments on curious-looking line profiles, A\&AS, 95, 41

Dunkin, S. K., Barlow, M. J., \& Ryan, S. G., 1997, High-resolution spectroscopy of Vega-like stars - I. Effective temperatures, gravities and photospheric abundances, MNRAS, 286, 604

Edvardsson, B., Andersen, J., Gustafsson, B., Lambert, D. L., Nissen, P. E., \& Tomkin, J., 1993, The Chemical Evolution of the Galactic Disk - Part One - Analysis and Results, A\&A, 275, 101

ESA 1997, The Hipparcos and Tycho Catalogues, ESA SP-1200, available from NASA-ADC or CDS in a machine-readable form (file name: hip_main.dat)

Flower, P. J., 1996, Transformations from Theoretical Hertzsprung-Russell Diagrams to Color-Magnitude Diagrams: Effective Temperatures, B-V Colors, and Bolometric Corrections, ApJ, 469, 355
Fossati, L., Bagnulo, S., Landstreet, J., Wade, G. Kochukhov, O., Monier, R., Weiss, W., \& Gebran, M., 2008, The effect of rotation on the abundances of the chemical elements of the A-type stars in the Praesepe cluster, A\&A, 483, 891

Gebran, M., Monier, R., \& Richard, O., 2008, Chemical composition of $\mathrm{A}$ and $\mathrm{F}$ dwarf members of the Coma Berenices open cluster, A\&A, 479, 189

Gebran, M. \& Monier, R., 2007, in Convection in Astrophysics, ed. F. Kupka, I. W. Roxburgh, \& K. L. Chan, Proc. IAU Symp. 239 (Cambridge: Cambridge University Press), 160

Gebran, M. \& Monier, R., 2008, Chemical composition of $\mathrm{A}$ and $\mathrm{F}$ dwarfs members of the Pleiades open cluster, A\&A, 483, 567

Gies, D. R. \& Lambert, D. L., 1992, Carbon, nitrogen, and oxygen abundances in early B-type stars, ApJ, 387,673

Girardi, L., Bressan, A., Bertelli, G., \& Chiosi, C., 2000, Evolutionary tracks and isochrones for lowand intermediate-mass stars: From 0.15 to $7 \mathrm{M}_{\odot}$, and from $\mathrm{Z}=0.0004$ to 0.03 , A\&AS, 141, 371

Hauck, B. \& Mermilliod, M., 1980, Uvbybeta photoelectric photometric catalogue, A\&AS, 40, 1

Kang, D.-I., Park, H.-S., Han, I.-W., Valyavin, G., Lee, B.-C., \& Kim, K.-M., 2006, Development Of An Automatic Processing Program for Boes Data II, PKAS, 21, 101

Kato, K., \& Sadakane, K. 1982, A model atmosphere analysis of Procyon ( $\alpha \mathrm{CMi}, \mathrm{F} 5 \mathrm{IV}-\mathrm{V}), \mathrm{A} \& \mathrm{~A}, 113$, 135

Kilian, J., 1992, Chemical abundances in early B-type stars. IV - He, CNO, and SI abundances, A\&A, 262, 171

Kilian, J., 1994, Chemical abundances in early B-type stars V. Metal abundances and LTE/NLTE comparison, A\&A, 282, 867

Kurucz, R. L., 1993, Kurucz CD-ROM, No. 13 (Harvard-Smithsonian Center for Astrophysics)

Kurucz, R. L. \& Bell, B., 1995, Kurucz CD-ROM, No. 23 (Harvard-Smithsonian Center for Astrophysics)

Kurucz, R. L., \& Peytremann, E., 1975, Smithsonian Astrophys. Obs. Spec. Rept., No. 362

Lemke, M., 1990, in The Atmospheres of EarlyType Stars, ed. U. Heber \& C. S. Jeffery (Berlin: Springer), p.54

Lemke, M., 1993, in Peculiar versus Normal Phenomena in A-type and Related Stars, eds. M.M. Dworetsky, F. Castelli, \& R. Faraggiana (San Francisco: Astronomical Society of the Pacific), p.407

Luck, R. E. \& Lambert, D. L., 1985, Carbon, nitrogen, and oxygen in intermediate-mass supergiants Is oxygen underabundant?, ApJ, 298, 782 
Moon, T. T., 1985, Commun. Univ. London Obs., No. 78

Napiwotzki, R., Schönberner, D., \& Wenske, V., 1993, On the determination of effective temperature and surface gravity of B, A, and F stars using Stromgren UVBY beta photometry, A\&A, 268, 653

Niemczura, E., 2003, Metallicities of the SPB stars from the IUE ultraviolet spectra, A\&A, 404, 689

Nissen, P. E., 1993, in Inside the stars, ASP Conf. Ser. 40, eds. W. W. Weiss \& A. Baglin, (San Francisco: Astronomical Society of the Pacific), p. 108

Royer, F., Gerbaldi, M., Faraggiana, R., \& Gómez, A. E., 2002a, Rotational velocities of A-type stars. I. Measurement of $\mathrm{v}$ sin $\mathrm{i}$ in the southern hemisphere, A\&A, 381, 105

Royer, F., Grenier, S., Baylac, M.-O., Gómez, A. E., \& Zorec, J. 2002b, Rotational velocities of A-type stars in the northern hemisphere. II. Measurement of $\mathrm{v} \sin \mathrm{i}, \mathrm{A} \& \mathrm{~A}, 393,897$

Sadakane, K., 1990, in Accuracy of element abundances from stellar atmospheres, Lecture Note in Physics, No. 356, ed. R. Wehrse (Berlin and New York: Springer), 57

Sadakane, K. \& Okyudo, M. 1989, Abundances of nitrogen and sulfur in A-type stars, PASJ, 41, 1055

Savanov, I. S., 1995a, Abundance anomalies in the atmospheres of rapidly-rotating Am stars, Astron. Rep., 39, 653

Savanov, I. S., 1995b, On the negative correlation between the $[\mathrm{C} / \mathrm{Fe}],[\mathrm{N} / \mathrm{Fe}],[\mathrm{O} / \mathrm{Fe}]$, and $\mathrm{Fe}$ abundances in the atmospheres of upper-main-sequence chemically-peculiar stars, Astron. Lett., 21, 684

Steffen, M., 1985, A model atmosphere analysis of the F5 IV-V subgiant Procyon, A\&AS, 59, 403

Takeda, Y., 1995, Self-consistent multi-parameter fitting of stellar flux spectra, PASJ, 47, 287

Takeda, Y., 2003, Oxygen line formation in late-F through early-K disk/halo stars. Infrared O I triplet and [O I] lines, A\&A, 402, 343

Takeda, Y., et al., 2005a, High-Dispersion Spectra Collection of Nearby F-K Stars at Okayama Astrophysical Observatory: A Basis for Spectroscopic Abundance Standards, PASJ, 57, 13

Takeda, Y. \& Honda, S., 2005, Photospheric CNO Abundances of Solar-Type Stars, PASJ, 57, 65

Takeda, Y., Ohkubo, M., Sato, B., Kambe, E., \& Sadakane, K., 2005b, Spectroscopic Study on the Atmospheric Parameters of Nearby F-K Dwarfs and Subgiants, PASJ, 57, 27 [Erratum: PASJ, 57, 415]

Takeda, Y. \& Sadakane, K., 1997, Oxygen $\lambda 7771-5$ Triplet in Hyades A-Type Stars: A Quest for the Key to the AM Phenomenon, PASJ, 49, 367

Takeda, Y., Sato, B., \& Murata, D., 2008, Stellar Parameters and Elemental Abundances of Late-G Giants, PASJ, 60, 781
Takeda, Y. \& Takada-Hidai, M., 1998, Oxygen Abundances in Late-B through F Supergiants, PASJ, 50, 629

Takeda, Y., Takada-Hidai, M., Jugaku, J., Sakaue, A., \& Sadakane, K. 1999, Oxygen 6156-8 Angstroms Triplet in Chemically Peculiar Stars of the Upper Main Sequence: Do HgMn Stars Show an Oxygen Anomaly?, PASJ, 51, 961

Varenne, O. \& Monier, R., 1999, Chemical abundances of A and F-type stars: the Hyades open cluster, A\&A, 351, 247

Young, P. R., 2005, The Ne/O abundance ratio in the quiet Sun, A\&A, 444, L45 